\title{
Effects of Aging and Display Contrast on the Legibility of Characters on Mobile Phone Screens
}

\author{
Satoshi Hasegawa ${ }^{1}$, Masaru Miyao ${ }^{2}$, Shohei Matsunuma ${ }^{3}$, Kazuhiro Fujikake ${ }^{4}$ and Masako Omori ${ }^{5}$ \\ ${ }^{1}$ Nagoya Bunri University, Aichi, Japan \\ 2 Nagoya University, Nagoya, Japan \\ ${ }^{3}$ Nagoya Institute of Technology, Nagoya, Japan \\ ${ }^{4}$ Institute for Science of Labour, Kawasaki, Japan \\ ${ }^{5}$ Kobe Women's University, Kobe, Japan
}

\begin{abstract}
Nowadays, mobile phones are very popular in the world. The use of text email with short message service (SMS) is spreading. In this study, we evaluated the legibility of characters on the liquid crystal displays of mobile phones. When characters are small, younger people assure readability by shortening the viewing distance. However, elderly people find it far more difficult to see small characters. Moreover, legibility deteriorates as the contrast of display becomes lower.
\end{abstract}

Index Terms — character size, elderly person, LCD, visibility

\section{INTRODUCTION}

The number of users for mobile phones is more than 2 billion worldwide. The use of text email with short message service (SMS) is spreading, and the number of occasions in which text information is handled through Web access from mobile phones is increasing.

Mobile phones have become the information terminal that is often nearest to us in our daily lives, and today they are used to distribute text information including disaster information [1] and living information. Mobile phones are widespread and commonly available, and should be easy to use for everyone, including the elderly [2-4]. We have been evaluating the readability of characters on mobile phones by the experiment of reading aloud the short sentence in Japanese or English [4, 5]. In this study, we evaluated the legibility of characters on the liquid crystal displays of mobile phones, with an original method in which we used the meaningless alphanumerical sentences

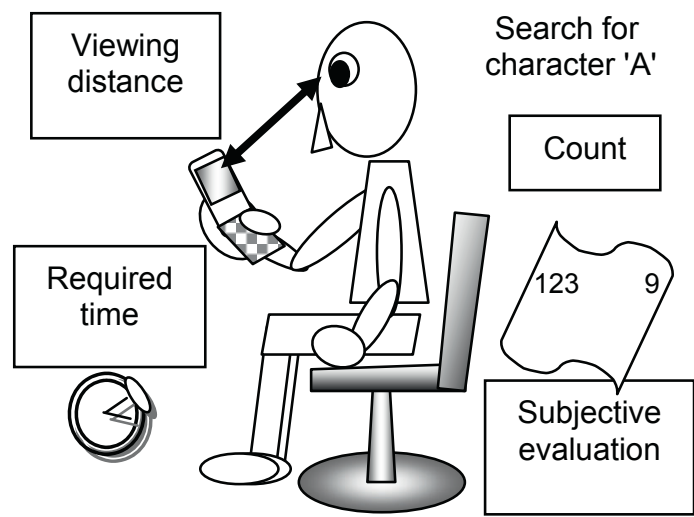

Figure 1. Experimental setup and measured items that were modified for mobile phones from the methods of ISO standards for display evaluation $[6,7]$.

\section{METHOD}

A method for evaluating flat panel displays is highlighted in ISO 9241-3. Amendment 1 [6] was modified for mobile phones and was used for the purpose of investigating the legibility of characters displayed on mobile phones (Fig. 1). Subjects search meaningless alphanumeric text (Fig. 2 and Fig. 3) displayed on mobile phones using graphic format, for target characters.

We conducted two experiments in this study. Experiment 1 is to research the aging effects on the legibility, and experiment 2 is for the confirmation of the effects of the display contrast.

Three different sizes of text were used ( 3 heights for single characters, L: $2.5 \mathrm{~mm}, \mathrm{M}: 2.0 \mathrm{~mm}, \mathrm{~S}: 1.0 \mathrm{~mm}$. The width of the characters was $1 / 2$ the height in all cases) as shown in Fig. 2 and Fig. 3. The displayed text was meaningless, and with all character sizes the screen contained 200 characters: 20 (characters/line) $\times 10$ (lines) in the experiment 1 (Fig. 2), and 240 characters: $20 \times 12$, $30 \times 8,40 \times 6$ in size L, M, S in the experiment 2, respectively (Fig. 3).

We used a mobile phone (Panasonic P903iX) with a $240 \times 320$ dot (QVGA) liquid crystal display for the experiment 1 , on which the measured luminance in the middle brightness mode used in the experiment was 116.0 $\pm 2.1 \mathrm{~cd} / \mathrm{m}^{2}$, and the contrast ratio was 19.3. Mobile phone used in the experiment 2 (Kyocera W43K) had the same size display, however, the contrast was varied as shown in Table 1. Four types of contrast of W, X, Y, and Z were prepared by using graphic text of PNG format (Fig. 3, Table 1).

\begin{tabular}{|c|c|c|}
\hline 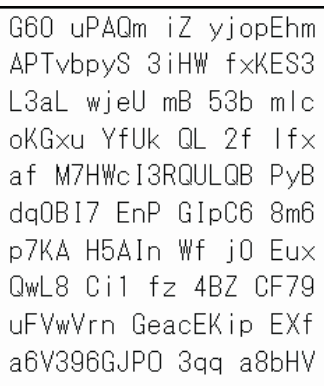 & 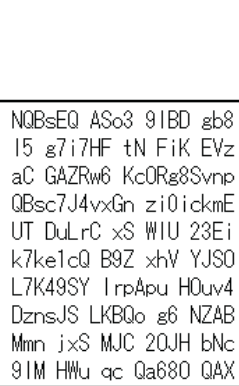 & 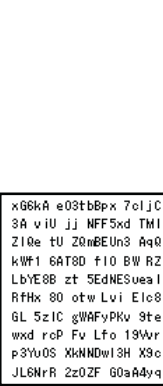 \\
\hline & $\mathrm{M}$ & S \\
\hline
\end{tabular}

Figure 2. Examples of meaningless text used in the experiment 1 


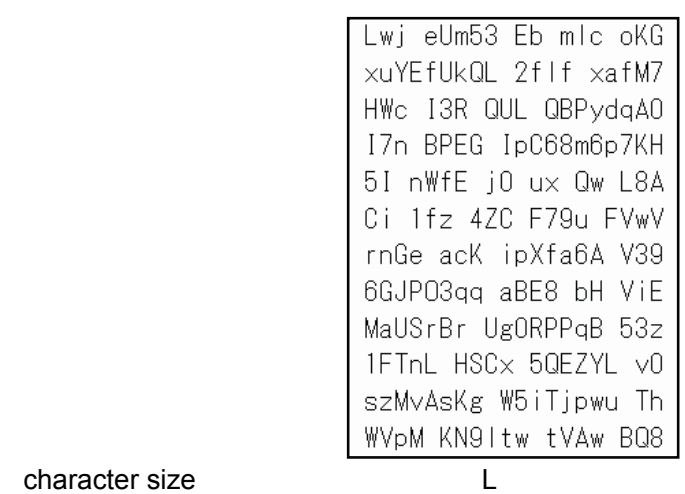

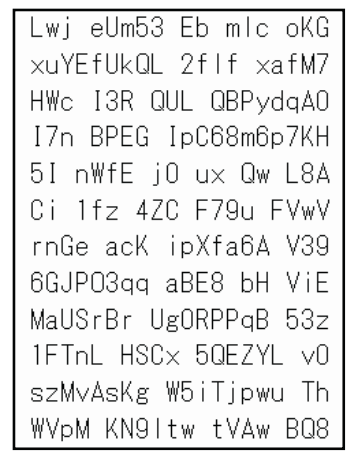

$\mathrm{X}$

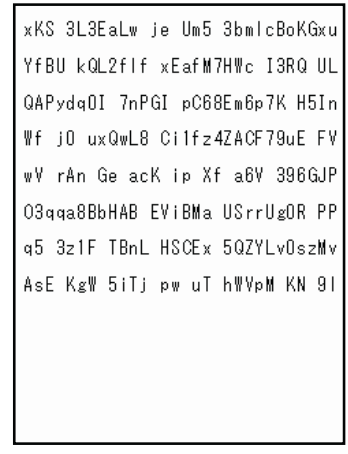

M
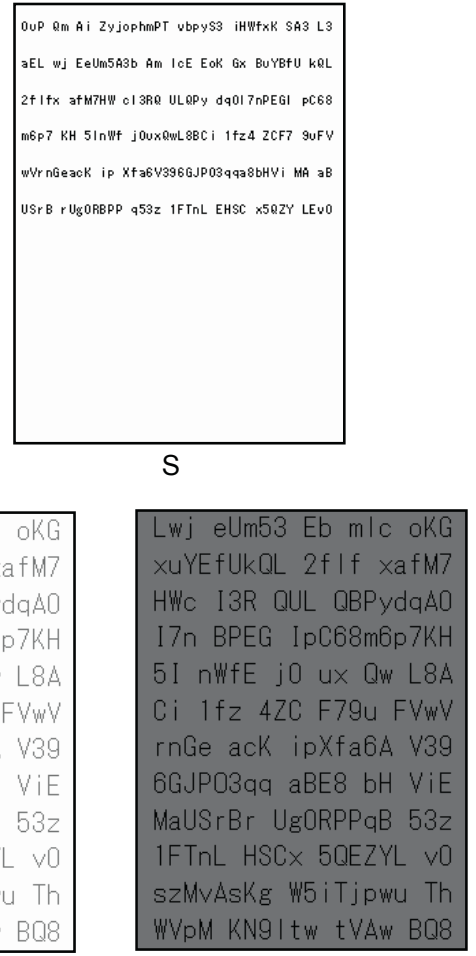

Z

Figure 3. Examples of meaningless text used in the experiment 2

TABLE I.

LUMINOSITY AND CONTRAST OF THE GRAPHIC TEXT USED IN THE EXPERIMENT 2.

\begin{tabular}{|c|l|l|l|l|}
\hline Code & Character color & Background color & Background luminosity (cd/m $\left.\mathbf{m}^{2}\right)$ & Contrast ratio \\
\hline W & white & black & $3.2 \pm 0.0$ & 53.8 \\
X & black & white & $171.4 \pm 1.2$ & 53.8 \\
Y & gray & white & $171.0 \pm 1.8$ & 4.4 \\
Z & black & gray & $38.2 \pm 0.8$ & 12.2 \\
\hline
\end{tabular}

The subjects searched for target characters of "A" included in the text by following the character strings horizontally as if reading. In each experiment we measured the number of target characters detected, the time required for the search, the viewing distance (distance from eyes to display, that have been measured without fixing every after searching), and the subjective evaluation of the legibility from 1 (difficult to read) to 9 (easy to read) as shown in Fig. 1.

The subjects for experiment $1(\mathrm{n}=75)$ were Japanese men and women aged from 19 to 80 years $(42.5 \pm 18.2$ years), including 37 people aged 19-39 years (young group), 19 aged 40-59 years (middle-aged group), and 19 aged 60-80 years (elderly group). The subjects for experiment $2(n=78)$ were also of various ages $(19-76$, $39.9 \pm 17.1$ years). Subjects who normally wore glasses when reading the newspaper participated in the experiment wearing glasses as usual.

\section{RESULTS}

\section{A. Results of the experiment 1}

Age dependency of the (a) viewing distance, (b) search speed $=200$ (characters) $/$ required time (sec.), and (c) error rate $=|\mathrm{Nc}-\mathrm{Nt}| / \mathrm{Nt} \times 100(\%)$, where $\mathrm{Nc}$ is a count and $\mathrm{Nt}$ is a number of target characters, measured in the experiment 1are shown in Fig. 4.

Results of experiment 1 are also shown for each age group in Fig. 5 for the (a) subjective evaluation, (b) viewing distance, (c) search speed, and (d) error rate.

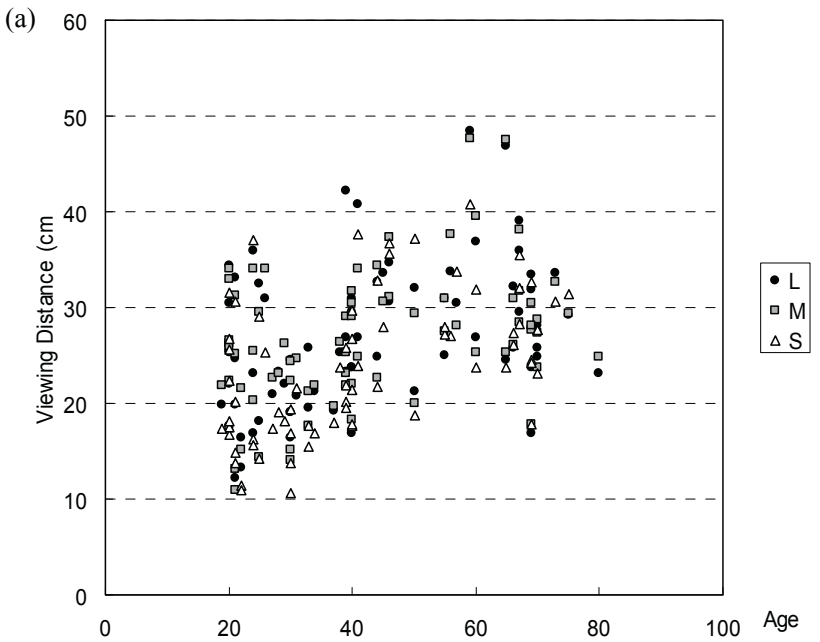

Figure 4. Age dependency of the results in experiment 1. (a) Viewing distance. Figure (b) and (c) are shown on the next page. 
(b)

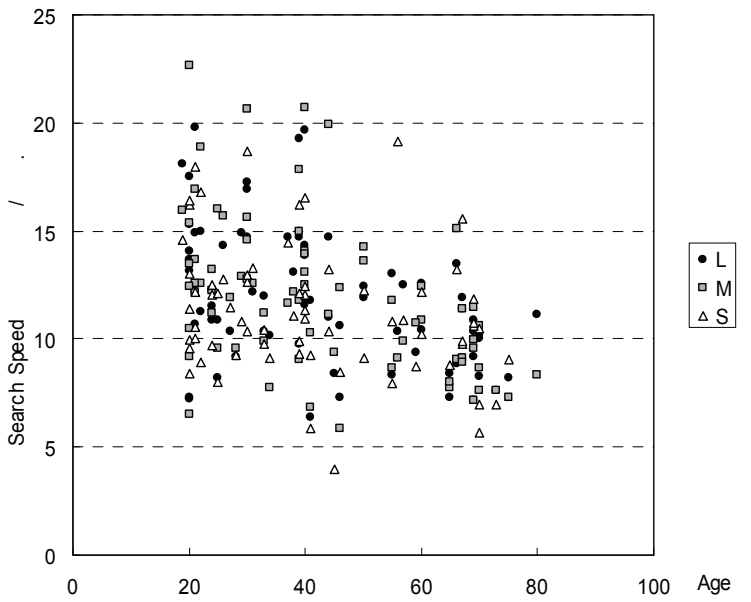

(c)

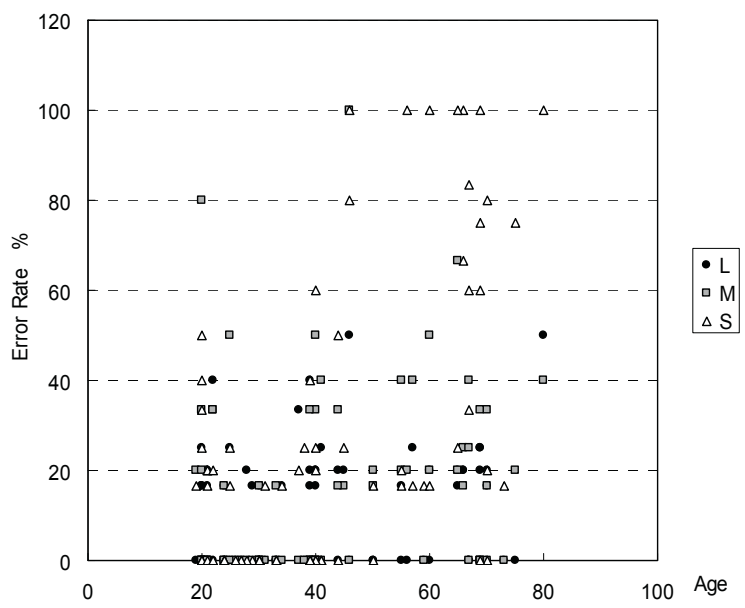

Figure 4. Age dependency of the results in experiment 1. (b) Search speed and (c) Error rate.

(a)

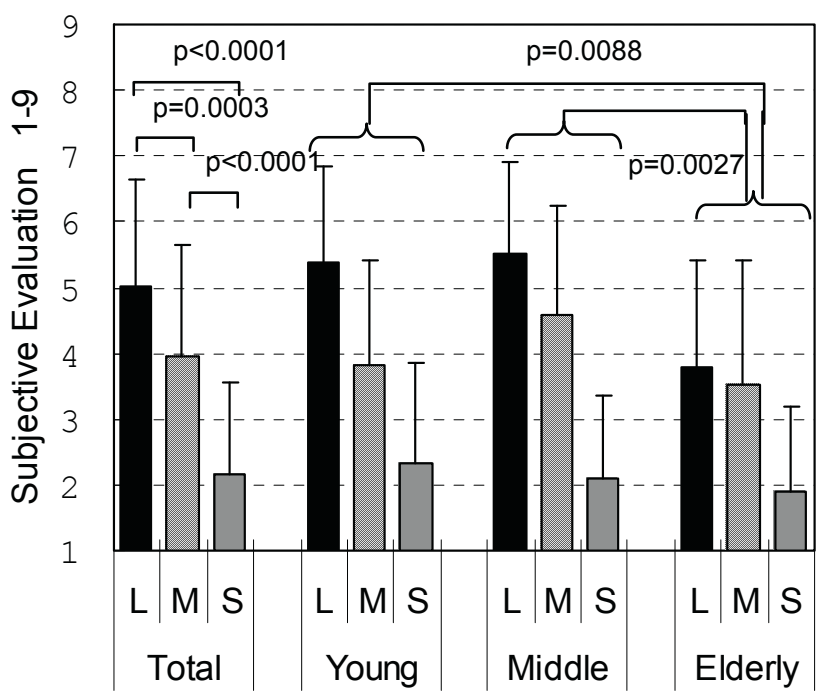

(c)

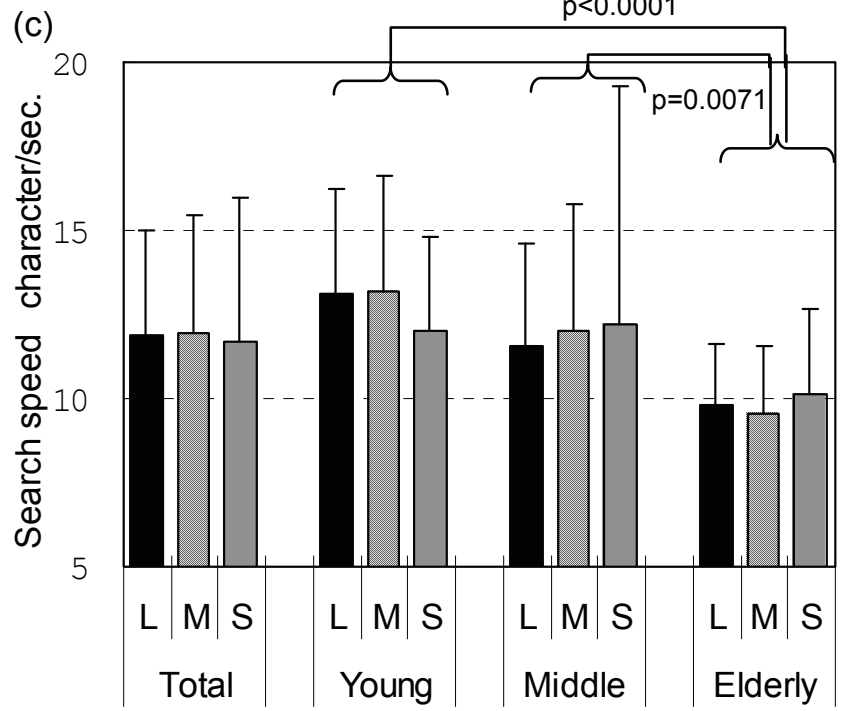

(b)

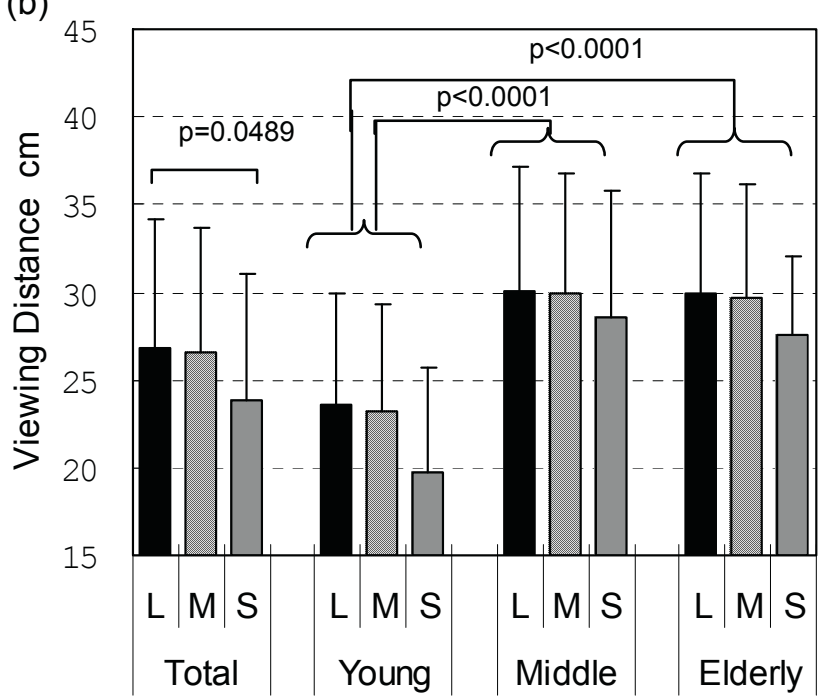

(d)

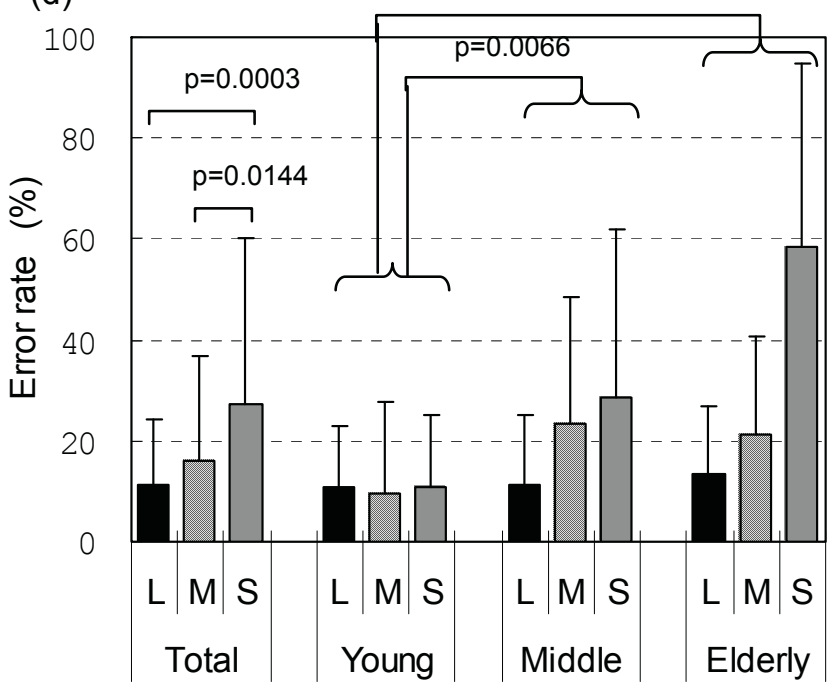

Figure 5. Results of experiment 1 for each age group of young: 19-39 years, middle-aged: 40-59 years, and elderly: 60-80 years 
A comparison of character size for all subjects (Fig. 5 (a)(d)) revealed that: (a) there was a significant decrease in the subjective evaluation in the order of character size $\mathrm{L}>$ $\mathrm{M}>\mathrm{S}$, where ' $>$ ' means the order of legibility, (b) viewing distance became shorter with character size $S$, (c) there was no significant difference in search speed with character size, and (d) the error rate was significantly higher with character size $\mathrm{S}$ than with the other sizes.

In the age dependency (Fig. 4) and the comparison between the age groups ("young," "middle-aged, "elderly," in Fig. 5 (a)-(d)) it was found that the elderly subjects had significantly lower subjective evaluation and search speed than the other groups. Meanwhile, the young

(a) Subjective evaluation
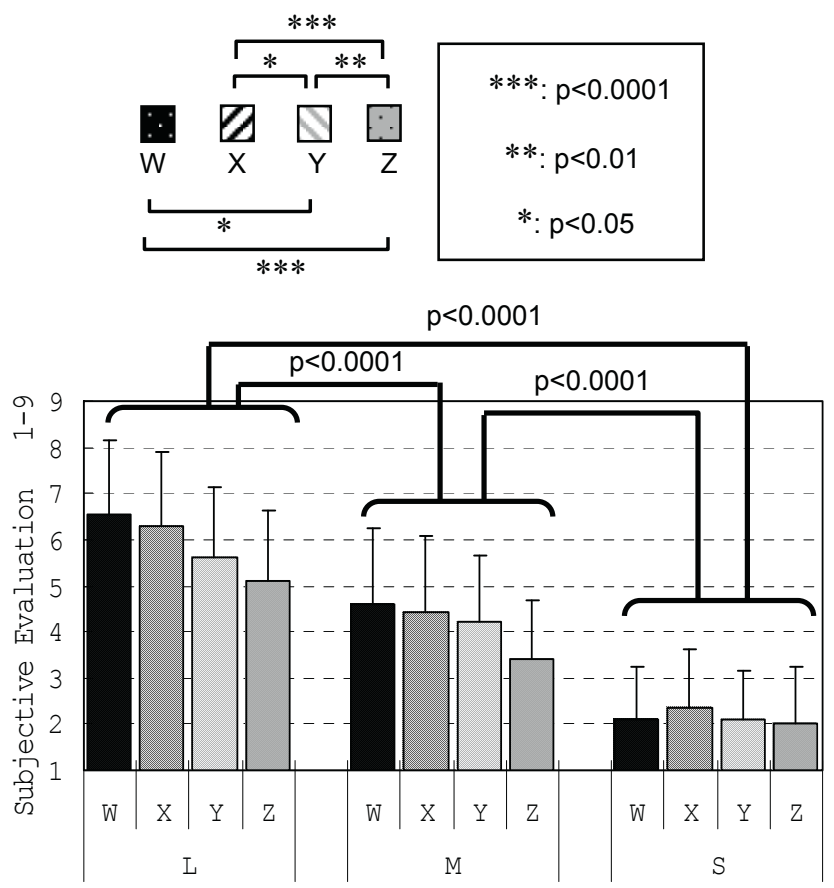

(c) Searching speed

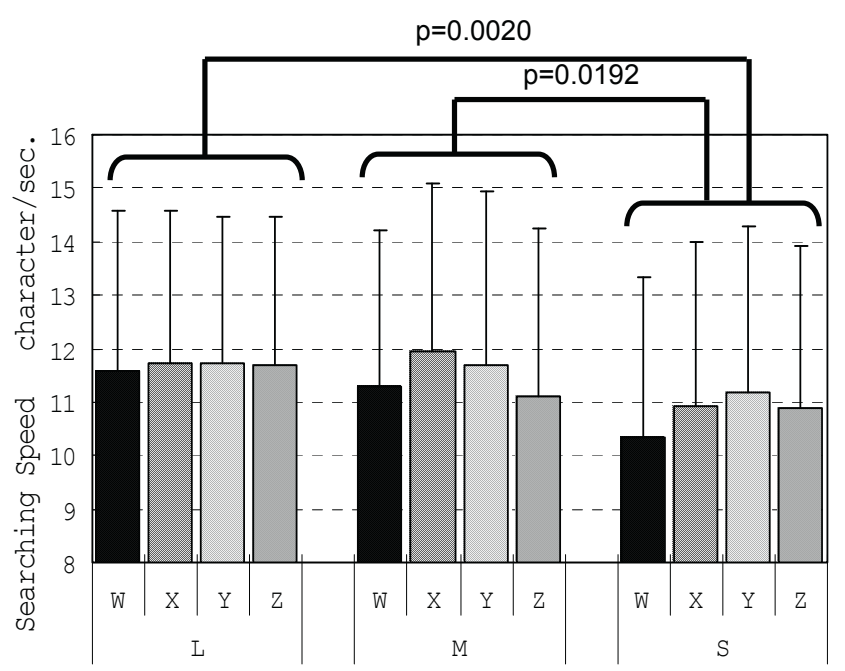

group had significantly shorter viewing distance and lower error rate.

\section{B. Results of the experiment 2}

Results of the experiment 2 are shown in Fig. 6 by the parameters of (a) subjective evaluation, (b) viewing distance, (c) searching speed $=240$ (characters) $/$ required time (sec.), and (d) error rate.

Fig. 6 shows that legibility was higher with larger characters $(\mathrm{L}>\mathrm{M}>\mathrm{S})$ agree with the result of experiment 1. Subjective evaluation, searching speed, and viewing distance decreased, and error rate increased with a decrease in the character size.

(b) Viewing distance

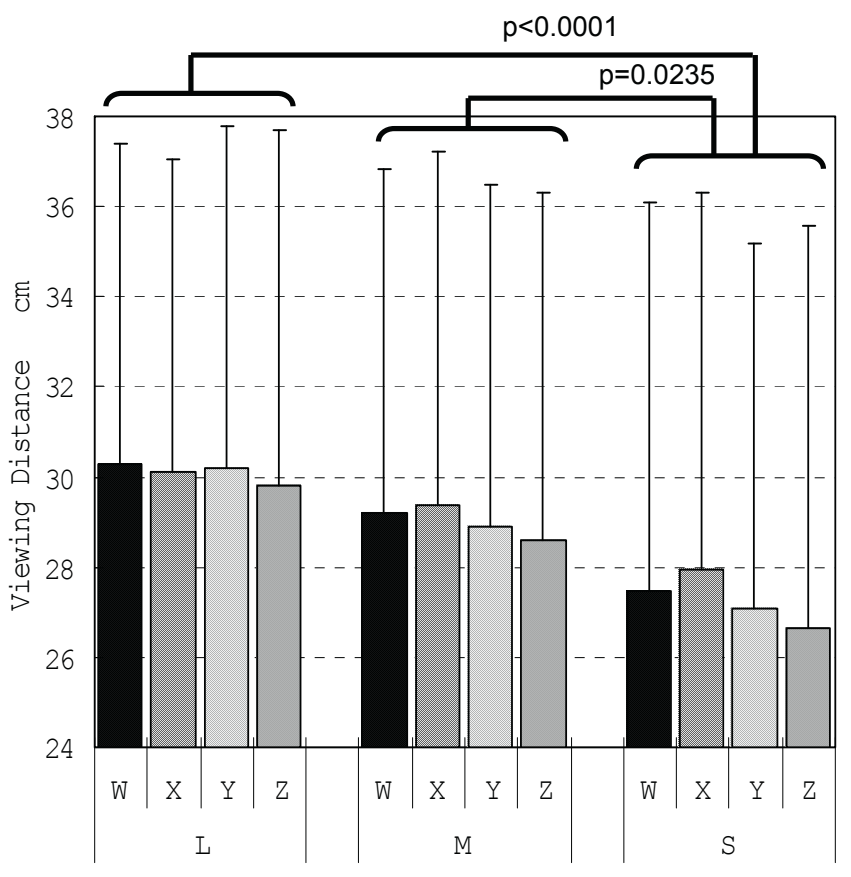

(d) Error rate

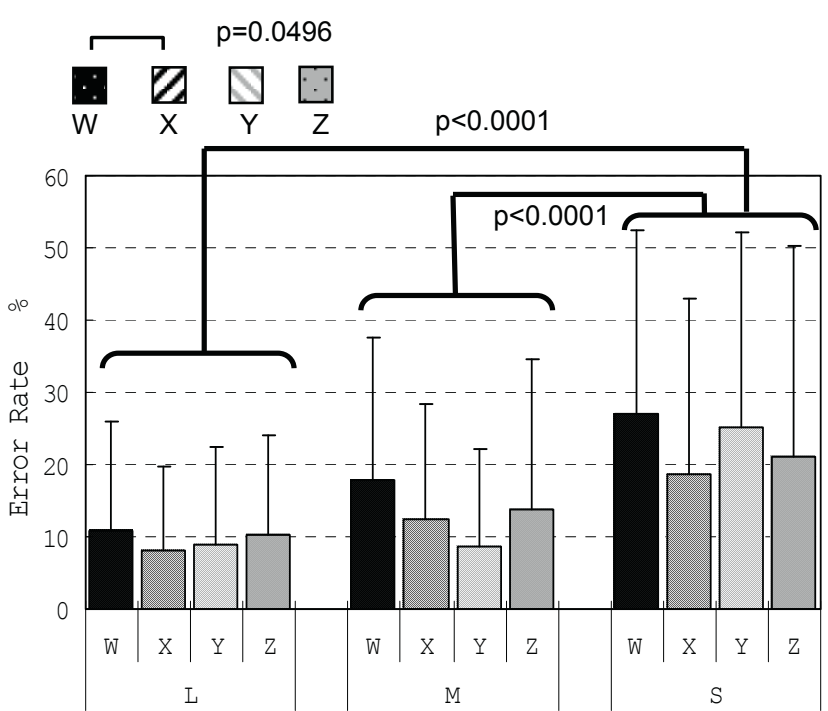

Figure 5. Results of experiment 2. Character size (L, M, S) and display contrast (W, X, Y, Z) are shown in Fig. 3 and Table 1. 
Subjective evaluation (Fig. 6(a)) shows $\mathrm{W}>\mathrm{X}>\mathrm{Y}>\mathrm{Z}$ although $\mathrm{W}>\mathrm{X}$ is not significant. However, objective parameter of error rate (Fig. 6(d)) shows that $X$ had higher readability than $\mathrm{W}$.

\section{DISCUSSION}

As VDT work criteria [8], the ISO 9241-3 (1992) [7] recommends that the minimum alphabetical or numerical character height should be 16 minutes of the arc, with character heights of 20 to 22 minutes of the arc preferred. The visual angle of 20-22 minutes of the arc corresponds to an assumed character height of $1.9 \mathrm{~mm}(\mathrm{M}$ size in this experiment) at a viewing distance of $30 \mathrm{~cm}$ for mobile phones.

The decrease in legibility to a decrease in the character size was shown in both experiment 1 and 2 with slightly different mobile phones. The effect by a decrease of the character size was objectively reflected by an increase in error rate in the aged group. In the young group, viewing distance decreased with a decrease in the character size, which may increase the visual angle, increasing the size of the displayed small characters on the retina for the maintenance of legibility. Viewing distance increased with the age of the subjects due to the influences of presbyopia $[2,4]$. The aged may have difficulty in adjusting viewing distance to optimal viewing distance, at least in the range allowing adjustment by the length of their arm with a mobile phone in their hand.

Since the experimental conditions are not equal, results of the experiment 1 and 2 cannot be compared easily. Nonetheless, the result of experiment 1 is well consistent with the result of type $\mathrm{Z}$ of the experiment 2 that have the nearest contrast ratio each other. Contrast ratio as well as character size would be important factor of the legibility. Legibility was lower with lower contrast ratio display, especially with smaller characters. Result of the objective evaluation in the experiment 2 showed that positive display (X), in which characters are black and background is white, had higher readability than negative display (W) especially when the character size was small. Lower contrast ratio with gray characters in white background $(\mathrm{Y})$ or black characters in gray background $(\mathrm{Z})$ had lower legibility than the ordinary positive display.

Differences in the results between experiment 1 and 2 may be due to the difference of the number of characters displayed in one line that should have effect on the easiness of target character searching. Target searching method using meaningless sentences in this study is the objective way to evaluate legibility that is not influenced from the kind of language and/or the contents of the sentence. However, it possibly includes the artifacts indicated by Tamura [9] when subjects do not search characters sequentially as they read sentences without obeying the instruction unconsciously or inevitably. Subjective evaluation and viewing distance showed a similar tendency of the results by reading method using Japanese, English or other languages [1, 4, 5], however, searching speed does not show strong differences except age dependency, as it was shown in reading speed in our previous study using natural languages $[1,4,5]$. Influence of age, character size, and contrast ratio was significantly shown in the error rate of detected target characters in this method. It is notable that legibility of characters on mobile phone screen has been quantified by subjective and objective evaluation and the influences of character size, display contrast, and user's age were indicated definitely.

\section{CONCLUSION}

When characters on a mobile phone are small, younger people assure readability by shortening the viewing distance. Conversely, elderly people find it far more difficult to see small characters. They increased the viewing distance irrespective of the character size, although, it is not enough to increase legibility. Moreover, lower contrast ratio also deteriorates the legibility.

In the current ubiquitous society, mobile terminals should be usable and harmless for workers, students and citizens of all age groups with various visual conditions. Universal design that considers the abilities of older people is desirable.

Results of this study would contribute to show guidelines for mobile character terminals equal to VDT work standards [6, 7] that had been shown for conventional desktop type information.

\section{REFERENCES}

[1] S. Hasegawa, K. Sato, S. Matsunuma, M. Miyao, and K. Okamoto, "Multilingual disaster information system: Information delivery using graphic text for mobile phones," AI \& Society, 19(3), pp.265-278, 2005. doi:10.1007/s00146-005-0316-7

[2] M. Omori, T. Watanabe, J. Takai, H. Takada, M. Miyao, "Readability and characteristics of the mobile phones for elderly people." Behaviour \& Information Technology. 21, pp.313-6, 2002. doi:10.1080/0144929021000048466

[3] I. Darroch, J. Goodman, S. Brewster, and P. Gray, "The effect of age and font size on reading text on handheld computers." INTERACT 2005, LNCS 3585, pp.253-266, 2005.

[4] S. Hasegawa, S. Matsunuma, M. Omori, and M. Miyao, "Aging effects on the readability of graphic text on mobile phones," Gerontechnology, 4(4), pp.200-208, 2006.

[5] S. Hasegawa, K. Fujikake, M. Omori, and M. Miyao, "Readability of characters on mobile phone liquid crystal displays." International Journal of Occupational Safety and Ergonomics (JOSE), vol. 14, no.3, 2008. in press.

[6] ISO 9241-3. Amendment 1. International Organization for Standardization, 2000.

[7] ISO 9241-3. Ergonomic requirements for office work with visual display terminals (VDTs) \& \#8211; Part 3: Visual display requirements. International Organization for Standardization, 1992.

[8] M. Miyao, S. S. Hacisalihzade, J. S. Allen, and L. W. Stark, "Effect of VDT resolution on visual fatigue and readability: an eye movement approach." Ergonomics. 32, pp.603-14, 1989. doi:10.1080/00140138908966135

[9] H. Tamura, M. Omori, S. Hasegawa, K. Fujikake, and M. Choui, "NIRS quick component analysis of brain activities engaged in visual text search," Symposium on Mobile Interaction 2008, Tokyo, Japan. pp.187-190, 2008 (in Japanese).

\section{AUTHORS}

Satoshi Hasegawa (hasegawa@nagoya-bunri.ac.jp) is with the Faculty of Information Culture, Nagoya Bunri University, 365 Maeda Inazawa-cho, Inazawa, Aichi 4928520, Japan.

Masaru Miyao (mmiyao@med.nagoya-u.ac.jp) is with the Information Technology Center, Nagoya University, Furo-cho, Chikusa-Ku, Nagoya 464-8601, Japan.

Shohei Matsunuma is with the Nagoya Institute of Technology, \#Umezaki Lab., Gokiso-cho, Showa-ku, Nagoya 466-8555, Japan. 
Kazuhiro Fujikake (k.fujikake@isl.or.jp) is with the Institute for Science of Labour, 2-8-14 Sugao, Miyamaeku, Kawasaki 216-8501, Japan.

Masako Omori (masako@suma.kobe-wu.ac.jp) is with the Faculty of Home Economics, Kobe Women's
University, 2-1 Aoyama Higashisuma, Suma-ku, Kobe 654-8585, Japan.

This article was modified from a presentation at the 21st Symposium on Human Factors in Telecommunication (HFT 2008) in Kuala Lumpur, Malaysia, March 2008. Manuscript received 20 July 2008. Published as submitted by the authors. 